\title{
Subject Index to Volume 37
}

Abdominal pain, recurrent, celiac disease and, low incidence in The Netherlands, 213

Academic Bulletin Board, 512

Acidemia, 3-methylglutaconic, Smith-Lemli-Opitz syndrome and, 671

ACTH, see Adrenocorticotropic hormone Actin, developing stomach and, 202

Acyl-CoA dehydrogenase, deficiency, neonatal screening study in Pennsylvania, incidence and mutation frequency rates, 675

Adenosine, role, inhibiting nonshivering thermogenesis, fetal sheep, 303

Adolescent

glycoprotein syndrome type 1 , endocrinology of, 395

salivary cortisol levels, 502

Adrenal cortical function, salivary cortisol levels and, childhood through adolescence, 502

Adrenal insufficiency, plasma cortisol concentrations and, VLBW infants, 112

Adrenocorticotropic hormone growth-promoting actions, T-lymphoblast cell lines, 507

levels, interleukin- $1 \beta$ effects, infant and prepubertal rats, 714

Amine metabolism, biogenic, neurologic disorders and, during methotrexate therapy for leukemia, 151

Amino acid

metabolism, phenylalanine levels and, proton nuclear magnetic resonance spectroscopy analysis, 244

transport properties, during gestation, 571

Amniotic fluid, cytokine expression, reverse transcriptase polymerase chain reaction detection, 450

Angiotensin II, furosemide and, effects, kidney growth and development, rat, 747

Antiinflammatory drugs, nonsteroidal, role as antipyretics for infants, retinal prostaglandin level effects, 81

Antioxidant enzyme

activity, after hyperoxia, endotoxin and dexamethasone effects, preterm infants, 469

status, plasma copper and, children with Wilson's disease, 219

thyrotropin and dexamethasone effects, fetal rats, 611
Antithrombin III, thrombin regulation role, endothelial cell surfaces and, 373

Apnea, swallowing during, preterm infants, 796

Artery, see also specific type

myogenic response, fetal pulmonary hypertension and, 196

Arthritis, rheumatoid, immunologic development, tumor necrosis factor gene effects, autoimmunity and, 165

Ascorbic acid, fruit drink containing, iron bioavailability measurement and, infant weaning foods, 389

Asphyxia

cerebral energy failure and, hypothermia effects on, neonatal piglet, 667

fetal, cardiovascular system and CNS effects, 707

glial fibrillary acidic protein levels in cerebrospinal fluid, role, assessing, neonates, 260

perinatal, effects on central nervous system function, rats, 489

somatosensory evoked potentials and brain water content after, preterm piglets, 661

Ataxia, NARP syndrome and, clinical symptoms relation to proportion of DNA carrying point mutation, 634

Atrial natriuretic peptide

diastolic filling effects, chick embryo, 465

dietary sodium modulation, neonatal rats, 310

Autoimmunity

epidermal growth factor, induction of, rats, 169

tumor necrosis factor and, sequence analysis, 165

Autonomic nervous system, development and maturation, spectral analysis of heart rate fluctuations, 294

Benzo $[a]$ pyrene, neonatal administration, hepatic cytochrome P450 enzyme imprinting, adult rat, 646

Bile acids, synthesis, inborn error of, familial giant cell hepatitis and, infant, 424

Biopterin, neurologic disorders and, during methotrexate therapy for leukemia, 151

Bisferiens peaks, radial artery pressure wave, role, marker of patent ductus arteriosus, neonates, 800
Blood

cells, peripheral, fatty acid oxidation defects and, 354

hematopoietic progenitor cells, placental, hemoglobin expression after transplant, fetus, 432

oxygen, tension, inhaled nitric oxide effects on, preterm lambs, 35

sialyl Lewis ${ }^{\mathrm{a}}$ antigen levels, infants and children with cystic fibrosis, 460

Blood flow, cerebral, microvascular injury and, piglets, 10

Body

fat-free mass, evaluating, total body electrical conductivity measurement role, infants, 94

whole, protein turnover, $\left[{ }^{15} \mathrm{~N}\right]$ glycine and $\left[1-{ }^{13} \mathrm{C}\right]$ leucine administration and, preterm infants, 381

Bone marrow, G-CSF and neutrophil progenitors in, fetus, 806

Brain

adrenal medulla, mRNA encoding tyrosine hydroxylase, dopamine $\beta$-hydroxylase, neuropeptide tyrosine, and prepro-enkephalin up-regulation in, postnatal oxygenation and, 701

cell membrane function, during hypoxia, hyperglycemic neonatal piglets, 133

c-fos, tyrosine hydroxylase and neuropeptide mRNA expression in, hypoxia and hypothermia effects, rat, 15

metabolic assessment during development, proton magnetic resonance spectroscopy, HPLC, and gas chromatography analysis, 145

phenylalanine levels and, proton nuclear magnetic resonance spectroscopy analysis, 244

water content, somatosensory evoked potentials and, after asphyxia, preterm piglets, 661

Breast-feeding

celiac disease and, low incidence in The Netherlands, 213

dietary energy requirements of infant and, 239

Breathing, periodic, swallowing during, preterm infants, 796

Brush-border enzymes, development and control, small intestine, fetal pig, 207 
SUBJECT INDEX TO VOLUME 37

Calorimetry, indirect, energy expenditure measure, head injury effects, children, 409

Carbohydrate, deficiency, glycoprotein syndrome type 1 and, endocrinology of, birth through adolescence, 395

Cardiovascular drugs, effects on circulation and hemodynamics, chick embryo, 117

Cardiovascular system, perinatal asphyxia effects on, fetal sheep, 707

Catecholamines

adrenal, synthesis and release, splanchnic nerve regulation maturity, 701

chromogranin A and, levels at birth, mode of delivery effects on, 101

Catheter

central venous, infections related to, Staphylococcus epidermidis virulence, 70

esophageal, chest wall distortion and, effects, esophageal manometry, preterm infant, 617

Celiac disease, low incidence in The Netherlands, 213

Central nervous system

disorders, phenylalanine levels and, proton nuclear magnetic resonance spectroscopy analysis, 244

function, perinatal asphyxia effects on, rats, 489

leukotrienes and, biosynthesis and pathophysiologic significance, 1

perinatal asphyxia effects on, fetal sheep, 707

Cerebral energy failure/loss

cytochrome $a a_{3}$ reduction and, near-infrared spectroscopy measurement role in predicting, hypoxic piglets, 253

hypoxia-ischemia and, hypothermia effects on, neonatal piglet, 667

Cerebrospinal fluid

G-CSF levels in, aseptic meningitis and, 160

glial fibrillary acidic protein levels, role, assessment of asphyxiated neonates, 260

Cesarean section, effects, chromogranin A and catecholamine levels at birth, 101

Chenodexoycholic acid, familial giant cell hepatitis effects, infant, 424

Chest wall distortion, esophageal catheter position and, preterm infants on manometry, 617

Children

malnourished, protein-energy, osteocalcin regulation and, 606

prepubertal, chronic renal failure, spontaneous nocturnal growth hormone secretion and, 86
Cholecystokinin, somatostatin and, plasma levels, preterm infants and mothers at birth, 771

Cholestasis, intrahepatic, familial giant cell hepatitis and, inborn error of bile acid synthesis, infant, 424

Cholic acid, familial giant cell hepatitis effects, infant, 424

Chromatography, high-performance liquid, brain, metabolic assessment during development, 145

Chromogranin A, catecholamine, levels at birth, mode of delivery effects on, 101

Circulation, pulmonary, inhaled nitric oxide effects, preterm lambs, 35

Cognitive function

congenital hypothyroidism, children, 736

impairment, NARP syndrome and, clinical symptoms relation to proportion of DNA carrying point mutation, 634

Colostrum, gastrointestinal tract and liver growth effects, neonatal pigs, 593

Congenital malformations, diabetic pregnancy and, rats, 343

Constipation, celiac disease and, low incidence in The Netherlands, 213

Copper, plasma, antioxidant status and, children with Wilson's disease, 219

Corticosterone, levels, interleukin- $1 \beta$ effects, infant and prepubertal rats, 714

Cortisol

gene expression of renin-angiotensin system effects, fetal sheep, 741

hemorrhage, renin response and gene expression to, fetal lambs, 316

plasma concentration, gestational age, postnatal age, and illness effects, preterm infants, 112

salivary, levels through childhood and adolescence, 502

small intestine brush-border enzyme development and control and, fetal piglets, 207

Cystic fibrosis

hepatic glucose production, 600

serum sialyl Lewis ${ }^{\mathrm{a}}$ antigen levels, infants and children, 460

Cytochrome $a a_{3}$, reduction, near-infrared spectroscopy measurement, role, predicting cerebral energy loss, hypoxic piglets, 253

Cytochrome P450, hepatic, enzyme imprinting, by neonatal benzo[ $a]$ pyrene administration, adult rat, 646

Cytokine

expression, reverse transcriptase polymerase chain reaction detection, fetus, 450 phenotype and responder cell frequency, preterm infants stimulated by staphylococcal enterotoxin B, 455

production, $\mathrm{T}$ cells and, infant versus adult, 64

Cytotoxicity, nitric oxide-induced, glutathione protective role and, 41

Delivery, vaginal, versus cesarean section, effects, chromogranin $\mathrm{A}$ and catecholamine levels at birth, 101

Development, see also Growth

brain, metabolic assessment during, proton magnetic resonance spectroscopy, HPLC, and gas chromatography analysis, 145

deranged, diabetic pregnancy and, rats, 343

fetal

epidermal growth factor deficiency, induction of, rats, 169

epidermal growth factor deficiency effects, rats, 175

protein $\mathrm{C}$ and, ovine, 365

heart, diastolic ventricular filling and, chick embryo, 289

kidney, furosemide treatment and angiotensin II effects, rat, 747

spatial learning, perinatal asphyxia effects on, 489

stomach, actin and myosin levels, 202

Dexamethasone

lung antioxidant enzyme activity after hyperoxia effects, preterm infant, 469

thyrotropin and, effects, antioxidant enzyme activity, fetal rats, 611

Diabetes

mellitus, cystic fibrosis and, 600

pregnancy and, congenital malformations, rats, 343

zinc absorption, metallothionein and cysteine-rich intestinal protein role, rats, 321

Dietary energy, breast-feeding and, infants, 239

1,25-Dihydroxyvitamin $D_{3}$, calbindin- $D_{9 K}$ gene expression regulation and, rat chorioallantoic placenta, 720

Ductus arteriosus

closure, smooth muscle migration and, transforming growth factor $\beta_{1}$ inhibiting effects, fetal lamb, 561

patent, bisferiens peaks in radial artery pressure wave as marker of, neonates, 800

Dystrophins, deletion-containing, expression in $m d x$ muscle, gene therapy and dystrophin function implications, 693

ECMO, see Oxygenation, extracorporeal membrane

Edema, pulmonary, surfactant therapy effects, preterm infants, 265 
Electromyography, glottic closure during hypoxia, awake neonatal lambs, 482

Electroretinogram, nonsteroidal antiinflammatory drug alterations, neonate, 81

Embryo

atrial natriuretic peptide diastolic filling effects, chick, 465

diastolic ventricular filling, heart rate characteristics and, chick, 289

Enalapril, effects, kidney growth and development, rat, 747

Encephalomyopathy, necrotizing, NARP syndrome and, clinical symptoms relation to proportion of DNA carrying point mutation, 634

$\beta$-Endorphin, levels, interleukin- $1 \beta$ effects, infant and prepubertal rats, 714

Endothelial cell, $\alpha_{2}$-macroglobulin and antithrombin III role, thrombin regulation, 373

Endotoxin, lung antioxidant enzyme activity after hyperoxia effects, preterm infant, 469

Energy expenditure

cerebral, asphyxia and, hypothermia effects on, neonatal piglet, 667

whole body, severe head injury effects, hormonal mediators and, children, 409

Energy metabolism, myocardial, during pacing-induced changes in oxygen consumption, lambs, 182

Epidemiology, celiac disease, low incidence in The Netherlands, 213

Epidermal growth factor, biliary epithelial cells and, congenital polycystic kidney disease, 755

Epidermal growth factors, deficiency

effects during perinatal development, rats, 175

induction of autoimmunity, rats, 169

Epithelial cells

biliary, congenital polycystic kidney disease and, 755

M-CSF factor identification in, 437

Epithelial toxicity, alveolar, erythrocyte lysis and, pulmonary surfactant therapy effects on, 26

Escherichia coli, enteropathogenic, intestinal tract mucins and, 75

Evoked potentials, see specific types

Failure to thrive, HIV-infected, maternal-fetal interactions effects, 56

Fatty acids

acyl-CoA dehydrogenase deficiency and, neonatal screening study in Pennsylvania, incidence and mutation frequency rates, 675 oxidation, peripheral blood cell levels, 354

polyunsaturated, leukotrienes and, 1
Fetal alcohol syndrome, alcohol ingestion during pregnancy effects on heart vitamin A levels, fetal rats, 418

Fetus

chromogranin A and catecholamine levels at birth, mode of delivery effects on, 101

cytokine expression, reverse transcriptase polymerase chain reaction detection, 450

ductus arteriosus closure and smooth muscle cell migration, transforming growth factor $\beta_{1}$ inhibiting effects, lambs, 561

G-CSF and neutrophil progenitors in liver and bone marrow, 806

growth and development epidermal growth factor deficiency effects, rats, 175

epidermal growth factor deficiency induction, rats, 169

hemoglobin synthesis, ${ }^{\mathrm{G}} \gamma$ - and ${ }^{A} \gamma$-globins and, 361

hemorrhage, renin response and gene expression to, cortisol effects, lambs, 316

HIV-infected, maternal interactions with, effects, growth failure, 56

hypothyroidism, maternal-fetal transfer of thyroxine effects, rats, 497

maldevelopment, diabetic pregnancy and, rats, 343

nonshivering thermogenesis, adenosine inhibition role, sheep, 303

placental blood hematopoietic progenitor cell transplant, hemoglobin expression after, 432

placental glycine and leucine transport, 571

protein C development, ovine, 365

pulmonary hypertension, myogenic response of arterial vessels and, 196

renin-angiotensin system gene expression, cortisol effects, sheep

small intestine, brush-border enzyme development and control, piglets, 207

Fibroblasts, cultured skin, auto-fluorescence of, mucolipidosis 4 and, phenotypic marker identification, 687

Fibronectin, levels, developing lung, rabbits, 189

Fructose absorption, intestinal, dietary induction, weaning rats, 777

Furosemide, angiotensin II and, effects, kidney growth and development, rat, 747

Gastrointestinal tract

colostrum effects on growth, neonatal pigs, 593 differentiation, IGF-I and IGF-II effects, suckling rats, 586

disease, leukotrienes and, biosynthesis and pathophysiologic significance, 1

function, lecithin cholesterol acyl transferase activity and lipoprotein metabolism, preterm infants, 328

mucins, and enteropathogenic Escherichia coli, 75

G-CSF, see Granulocyte colony-stimulating factor

Gene expression

calbindin- $\mathrm{D}_{9 \mathrm{~K}}, 1,25$-dihydroxyvitamin $\mathrm{D}_{3}$ regulation of, chorioallantoic placenta, rats, 720

renin-angiotensin system, cortisol effects, fetal sheep, 741

translational control, 681

Gene therapy, deletion-containing dystrophin expression in $m d x$ muscle, 693

Genetic studies

$3 \beta$-hydroxysteroid dehydrogenase gene, molecular basis and hormonal criteria, pubarche children and hirsute female patients, 820

hypothyroidism, cognitive function and, children, 736

NARP syndrome, clinical symptoms and proportion of mitochondrial DNA carrying 8993 point mutation, 634

polycystic kidney disease, epithelial hyperplasia and, 755

Germ cell differentiation, mRNA translation control and, 681

${ }^{\mathrm{G}} \gamma$ - and ${ }^{\mathrm{A}} \gamma$-globins, hemoglobin synthesis and, preterm infants, 361

Glial fibrillary acidic protein, levels in cerebrospinal fluid, asphyxiated neonates, 260

Glottic closure, hypoxia and, neonatal lambs, 482

Glucose

hepatic production, children with cystic fibrosis, 600

turnover rate, alanine relationship, hepatic alanine metabolism during pregnancy and, rabbits, 764

Glutathione, protective role, nitric oxide-induced cytotoxicity, 41

Glycine, placental transport during pregnancy, 571

$\left[{ }^{15} \mathrm{~N}\right]$ Glycine, $\left[1-{ }^{13} \mathrm{C}\right]$ leucine administration and, effects, whole-body protein turnover, preterm infants, 381

Glycoprotein syndrome type 1 , carbohydrate-deficient, endocrinology of, birth through adolescence, 395

Granulocyte colony-stimulating factor cerebrospinal fluid levels, aseptic meningitis and, 160 
neutrophil pool sizes and, fetus, 806 recombinant human, hematopoietic progenitor cells effects, 630

Growth, see also Development

failure, maternal-fetal interactions and, HIV-1 infected mice, 56

gastrointestinal tract and liver, colostrum effects on, neonatal pigs, 593

lung, infant, protein deficiency effects on, 783

Growth hormone

deficiency, growth hormone-binding protein and IGF-I level normalization after therapy, children, 731

human, intestinal ion transport effects, rat, 576

recombinant human, plasma growth hormone-binding protein and IGF-I levels, Turner's syndrome, 106

spontaneous nocturnal secretion, chronic renal disease and, prepubertal children, 86

Haemophilus influenzae, nontypable, lymphotoxin effects, neutrophil-mediated killing, 155

Head injury, effects, whole body energy expenditure and, hormonal mediators and, children, 409

Heart

diastolic filling, atrial natriuretic peptide effects, chick embryo, 465

pacemaker derived from fetal rat myocardium, 283

Heart rate

diastolic ventricular filling and, chick embryo, 289

pacing-induced changes, myocardial energy metabolism during, neonatal lambs, 182

striatum damage and, asphyxia effects on, fetal sheep, 707

variability

artificial ventilation effects, very preterm infants, 124

spectral analysis of, autonomic nervous system development and maturation, 294

Hemodynamics, cardiovascular drugs, effects on circulation and hemodynamics, chick, 117

Hemoglobin

expression, after transplant of placental blood hematopoietic progenitor cells, fetus, 432

synthesis, ${ }^{\mathrm{G}} \gamma$ - and ${ }^{\mathrm{A}} \gamma$-globins and, preterm infants, 361

Hemorrhage

pulmonary, surfactant therapy effects on risk, VLBW infant with RDS, 26

renin response and gene expression, cortisol effects, fetal lambs, 316
Hemostatic system, protein $C$ development, ovine fetus, 365

Hepatitis, familial giant cell, bile acid synthesis and, infant, 424

HIV, see Human immunodeficiency virus

Hormones, see also specific types

mediators, severe head injury effects, whole body energy expenditure and, children, 409

HPLC, see Chromatography, high-performance liquid

Human immunodeficiency virus, maternal-fetal interactions effects, growth failure, 56

Hydrophobicity, skin surface, prenatal steroids effects, preterm rats, 402

$3 \beta$-Hydroxysteroid dehydrogenase, gene, molecular basis and hormonal criteria, pubarche children and hirsute female patients, 820

Hyperglycemia, brain cell membrane function during hypoxia, neonatal piglets, 133

Hyperoxia

antioxidant enzyme activity and, thyrotropin and dexamethasone effects, fetal rats, 611

lung antioxidant enzyme activity after, gene expression and endotoxin and dexamethasone treatment, preterm rabbits, 469

Hyperphenylalaninemia, phenylalanine levels and, proton nuclear magnetic resonance spectroscopy analysis, 244

Hyperplasia

congenital adrenal, $3 \beta$-hydroxysteroid dehydrogenase deficiency, molecular basis and hormonal criteria, 820

epithelial, congenital polycystic kidney disease and, 755

Hypertension

persistent pulmonary of newborn, urinary nitrite and nitrate concentrations, ECMO effects, 31

pulmonary, myogenic response of arterial vessels and, fetus, 196

Hypoglycemia, acyl-CoA dehydrogenase deficiency and, neonatal screening study in Pennsylvania, incidence and mutation frequency rates, 675

Hypotension, cerebral vasodilation and, endothelial injury effects, piglets, 10

Hypothermia

after asphyxia, effects on cerebral energy failure, neonatal piglet, 667

effects, c-fos, neuropeptide mRNA and tyrosine hydroxylase expression, rat brain around birth, 15
Hypothyroidism

congenital, neurophysiologic analysis and cognitive function, children, 736

in utero, maternal-fetal transfer of thyroxine effects, rat, 497

Hypoxia

brain cell membrane function during, hyperglycemic neonatal piglets, 133

cerebral energy loss and, cytochrome $a a_{3}$ reduction, near-infrared spectroscopy measurement role in predicting, piglets, 253

effects, c-fos, neuropeptide mRNA and tyrosine hydroxylase expression, rat brain around birth, 15

laryngeal response, neonatal lambs, 482 ventilatory response to, nicotine effects, lambs, 652

IGF, see Insulin-like growth factor

Immune system

interleukin-10 levels in human milk, 444

M-CSF identification in human milk and epithelial cells, 437

Immunity, interleukin-1 receptor antagonist, levels in neonatal sepsis, 626

Immunization, rubella, virus growth in vitro, cord blood mononuclear cells effects, 623

Immunoglobulin antibody, responses to pneumococcal polysaccharide vaccine, patients with recurrent respiratory tract infection, 812

Immunologic development, perinatal, tumor necrosis factor gene effects, autoimmunity and, 165

Incubator, water loss from skin, term and preterm infants, 233

Infant

body fat-free mass evaluations, total body electrical conductivity role, 94

breast-fed, dietary energy requirements and, 239

familial giant cell hepatitis, bile acid synthesis and, 424

glycoprotein syndrome type 1 , endocrinology of, 395

HIV-infected, growth failure and, maternal-fetal interactions effects, 56

interleukin- $1 \beta$ effects, $\mathrm{ACTH}$, $\beta$-endorphin, and corticosterone levels, rats, 714

lung growth, protein deficiency effects on, 783

M-CSF identification in human milk and epithelial cells, 437

nutrition

intestinal fructose absorption, dietary induction, weaning rats, 777 
SUBJECT INDEX TO VOLUME 37

weaning foods, iron bioavailability measurement and, 389

prenatally exposed to alcohol, heart vitamin A levels and, relation to fetal alcohol syndrome, rats, 418 prenatally exposed to nicotine, hyperventilatory response to hypoxemia and, SIDS relation, 652

preterm

cholecystokinin and somatostatin levels, at birth, 771

esophageal manometry, chest wall distortion effects on, 617

hemoglobin synthesis, ${ }^{\mathrm{G}} \gamma$ - and A $\gamma$-globins and, 361

hyperoxia, lung antioxidant enzyme activity after, gene expression and endotoxin and dexamethasone treatment, 469

IGF-I and IGF-binding protein, during first year, 581

inhaled nitric oxide effects on pulmonary circulation, lambs, 35

lipoprotein metabolism and, lecithin cholesterol acyl transferase activity, 328

lung vascular injury and edema, surfactant treatment effects, 265

plasma cortisol concentrations, gestational age, postnatal age, and illness effects, 112

prenatal steroids effects on skin surface hydrophobicity, rats, 402

swallowing during apnea and periodic breathing, comparison study, 796

visual development, pattern visual evoked potential evaluation, 140

water loss from skin, incubator and radiant heater and, 233

whole-body protein turnover, $\left[{ }^{15} \mathrm{~N}\right]$ glycine and $\left[1-{ }^{13} \mathrm{C}\right]$ leucine administration and, 381

respiratory failure, exogenous surfactant therapy, second dose distribution, rabbits, 476

stomach development, actin and myosin levels, 202

$T$ cells and cytokine expression, versus adult, 64

very low birth weight

intestinal fructose absorption, dietary induction, weaning rats, 777

RDS and, surfactant effects, pulmonary hemorrhage risk, 26

very preterm, artificial ventilation effects, heart rate variability, 124

Infection, catheter-related, Staphylococcus epidermidis virulence, 70

Insulin-like growth factor

gastrointestinal tract differentiation and, suckling rats, 586

plasma, maternal protein malnutrition effects, fetal rats, 334

Insulin-like growth factor-I
IGF-binding protein and, during furst levels year, term and preterm infants, 581

growth-hormone deficient children after therapy, 731

recombinant human growth hormone therapy effects, Turner's syndrome patients, 106

Interleukin-1 $\beta$, effects, plasma ACTH, $\beta$-endorphin, and corticosterone levels, infant and prepubertal rats, 714

Interleukin-10, levels in human milk, 444

Interleukin-1, receptor antagonist, levels in neonatal sepsis, 626

Intestinal protein, role, zinc absorption, diabetic rats, 321

Intestinal tract, mucins, and enteropathogenic Escherichia coli, 75

Intestine

ion transport, human growth hormone effects on, rat, 576

small, brush-border enzyme development and control, fetal pig, 207

Ion transport, intestinal, growth hormone effects on, rat, 576

Iron deficiency, bioavailability measurement in infant weaning foods, 389

Kidney

disease, congenital polycystic, epithelial hyperplasia and, 755

growth and development, furosemide treatment and angiotensin II effects, rat, 747

taurine transport regulation, medium osmolality effects, 227

Learning, spatial, perinatal asphyxia effects, rats, 489

Lecithin cholesterol acyl transferase, lipoprotein metabolism and, preterm infants, 328

Leucine acidemia and Smith-Lemli-Opitz syndrome, 671

placental transport during pregnancy, 571

Leukemia, methotrexate therapy, neurologic disorders and, biopterin and biogenic amine metabolism effects on, 151

Leukotrienes, biosynthesis, metabolism, and pathophysiologic significance, 1

Lipid storage, mucolipidosis 4 and, auto-fluorescence of cultured skin fibroblasts, phenotypic marker identification, 687 metabolism, 3-methylglutaconic
Lipoprotein metabolism, lecithin cholesterol acyl transferase activity and, preterm infants, 328

Liver

alanine metabolism, during pregnancy, glucose turnover rate and, rabbits, 764

colostrum effects on growth, neonatal pigs, 593

cytochrome P450 enzyme imprinting, neonatal administration of benzo[ $a]$ pyrene and, 646

damage, copper accumulation and, children with Wilson's disease, 219

function, familial giant cell hepatitis effects, inborn error of bile acid synthesis, infant, 424

G-CSF and neutrophil progenitors in, fetus, 806

glucose production, children with cystic fibrosis, 600

transplantation, tyrosinemia, type I nephropathy after, 640

Lung

development, fibronectin expression in, rabbits, 189

disease, leukotrienes and, biosynthesis and pathophysiologic significance, 1

failure, exogenous surfactant therapy, second dose distribution, rabbits, 476

growth, infant, protein deficiency effects on, 783

injury, surfactant treatment effects, preterm infants, 265

Lymphocytes, responder cell frequency, preterm infants stimulated by staphylococcal enterotoxin B, 455

Lymphotoxin, effects, neutrophil-mediated killing of nontypable Haemophilus influenzae, 155

$\alpha_{2}$-Macroglobulin, thrombin regulation role, endothelial cell surfaces and, 373

Macrophage colony-stimulating factor, identification in human milk and epithelial cells, 437

Malnutrition, protein-energy, osteocalcin regulation and, children, 606

Manometry, esophageal, chest wall distortion effects on, preterm infants, 617

Maternal protein, malnutrition, effects, fetal growth, rats, 334

Maternal-fetal interactions

cytokine expression, reverse transcriptase polymerase chain reaction detection, 450

failure to thrive, HIV-infected fetus, 56

thyroxine transfer, effects on in utero hypothyroidism, rats, 497 
M-CSF, see Macrophage colony-stimulating factor

$m d x$ muscle, deletion-containing dystrophin expression in, gene therapy and dystrophin function implications, 693

Medulla, adrenal, mRNA encoding tyrosine hydroxylase, dopamine $\beta$-hydroxylase, neuropeptide tyrosine, and prepro-enkephalin up-regulation, postnatal oxygenation and, 701

Meningitis, aseptic, G-CSF levels in cerebrospinal fluid and, 160

Metabolism, see also specific types hepatic alanine, plasma disposal rate and, pregnant rabbits, 764

inborn errors of, fatty acid oxidation defects and, 354

Metallothionein, role, zinc absorption, diabetic rats, 321

Methotrexate, leukemia therapy, neurologic disorders and, biopterin and biogenic amine metabolism effects on, 151

Microvascular injury, effects, arteriolar dilation, hypotensive piglets, 10

Milk

colustrum, gastrointestinal tract and liver, effects on growth, 593

human

interleukin-10 levels in, 444

M-CSF identification in, 437

production

protein deficiency effects, morphometric analysis, infant rats, 783

protein deficiency effects on, 783

Mitochondria

disorder, maternally inherited, clinical symptoms and proportion of mitochondrial DNA carrying 8993 point mutation, 634

renal tubular, $1,25(\mathrm{OH})_{2} \mathrm{D}$ production regulation, calcium attentuation, vitamin D-deprived rats, 726

Morphogenesis, tissue, fibronectin levels in developing lung, rabbits, 189

Morphometric analysis, protein deficiency effects, milk production and infant lung growth, 783

Motor disorders, fine, congenital hypothyroidism and, neurophysiologic analysis and cognitive function, 736

mRNA, see Ribonucleic acid, messenger

Mucolipidosis 4, auto-fluorescence of cultured skin fibroblasts, phenotypic marker identification, 687

Muscular dystrophy, Becker, dystrophin deficiency and, deletion-containing dystrophin expression $m d x$ muscle, gene therapy and dystrophin

function implications, 693

Myocardium, pacemaker derived from, fetal rat, 283

Myocytes, ventricular, triiodothyronine effects on $\beta$-adrenergic responsiveness retention, neonata rabbits, 277

Myogenic response, arterial vessels, fetal pulmonary hypertension and, 196

Myosin, developing stomach and, 202

NARP syndrome, see Neurogenic muscle weakness, ataxia, retinitis pigmentosa syndrome

Neonate

acyl-CoA dehydrogenase deficiency, screening study in Pennsylvania, incidence and mutation frequency rates, 675

asphyxia

effects on central nervous system function, rats, 489

glial fibrillary acidic protein levels in cerebrospinal fluid, role in assessing, 260

atrial natriuretic peptide, dietary sodium modulation, rats, 310

brain cell membrane function during hypoxia, hyperglycemic piglets, 133

gastrointestinal tract and liver, colustrum effects on growth, 593

hypoxia, glottic closure and, awake neonatal lambs, 482

oxygen consumption, pacing-induced changes, myocardial energy metabolism during, lambs, 182

patent ductus arteriosus, bisferiens peaks in radial artery pressure wave, role as marker, 800

retinal prostaglandin levels, nonsteroidal antiinflammatory drug effects on, 81

screening, congenital hypothyroidism, neurophysiologic analysis and cognitive function, 736

sepsis, interleukin-1 receptor antagonist levels, 626

ventricular myocyte culture, triiodothyronine effects on $\beta$-adrenergic responsiveness retention, 277

Nephropathy, tyrosinemia, type I, after liver transplantation, 640

Neurogenic muscle weakness, ataxia, retinitis pigmentosa syndrome, clinical symptoms relation to proportion of DNA carrying point mutation, 634

Neurologic impairment, glycoprotein syndrome type 1 and, endocrinology of, birth through adolescence, 395
Neurophysiologic analysis, congenital hypothyroidism, children, 736

Neutrophils

cerebrospinal fluid levels, during aseptic meningitis, 160

effects, nontypable Haemophilus influenzae killing, 155

G-CSF production and, liver and bone marrow, fetus, 806

Nitrate, concentrations, PPHN and ECMO effects, 31

Nitric oxide

cytotoxicity and, oxidative stress and glutathione protective role, 41

inhaled, effects, pulmonary circulation, preterm lambs, 35

Nitrite, urinary, concentrations, PPHN and ECMO effects, 31

Nutrition

breast-feeding and, energy requirements for infants, 239

infant weaning foods, iron bioavailability measurement and, 389

intestinal fructose absorption, weaning rats, 777

malnutrition, protein-energy, osteocalcin regulation, children, 606

maternal dietary protein malnutrition, effects, fetal growth, rats, 334

protein deficiency, effects, milk production and infant lung growth 783

whole body energy expenditure, severe head injury effects, children, 409

Nutritional assessment, fat-free mass evaluation, total body electrical conductivity measurement role, infants, 94

Osmolality, medium, taurine transport regulation and, 227

Osteocalcin, regulation, protein-energy malnutrition, children, 606

Otitis media, nontypable Haemophilus influenzae and, lymphotoxin and TNF effects on, 155

Oxidation

fatty acid, peripheral blood cell levels, role, detecting defects, 354

mitochondrial, fatty acids, acyl-CoA dehydrogenase deficiency and, neonatal screening study in Pennsylvania, incidence and mutation frequency rates, 675

Oxygen consumption, pacing-induced changes, myocardial energy metabolism during, neonatal lambs, 182

Oxygenation

extracorporeal membrane, effects, urinary nitrite and nitrate 
concentrations, persistent pulmonary hypertension of newborn, 31

postnatal, mRNA encoding tyrosine hydroxylase, dopamine

$\beta$-hydroxylase, neuropeptide tyrosine, and prepro-enkephalin up-regulation in adrenal medulla, 701

Pacemaker, derived from fetal rat myocardium, 283

Parathyroid hormone, growth-promoting actions, T-lymphoblast cell lines, 507

Pathophysiology, leukotrienes, 1

Pediatric Research, academic bulletin board, 512

Phenylalanine, levels in brain, proton nuclear magnetic resonance spectroscopy analysis, 244

Placenta, chorioallantoic, 1,25-dihydroxyvitamin $\mathrm{D}_{3}$ calbindin- $\mathrm{D}_{9 \mathrm{~K}}$ gene expression regulation and, rat, 720

Placental tissue, cytokine expression, reverse transcriptase polymerase chain reaction detection, 450

Plasma protein, inhibitory effects, surfactant activity, surfactant apoprotein A role, 21

Pneumotachography, glottic closure during hypoxia, awake neonatal lambs, 482

Polymerase chain reaction reverse transcriptase, fetal cytokine expression detection, 450

tumor necrosis factor gene analysis, autoimmunity and, 165

PPHN, see Hypertension, persistent pulmonary of newborn

Pregnancy alcohol ingestion during, effects, heart vitamin A levels, fetal alcohol syndrome and, rats, 418

diabetic, congenital malformations and, rats, 343

placental glycine and leucine transport during, 571

plasma disposal rate and hepatic alanine metabolism, rabbits, 764

Prostaglandin levels, retinal, nonsteroidal antiinflammatory drug effects on, neonates, 81

Protein

deficiency, effects on milk production and infant lung growth, 783

malnutrition, maternal, effects, fetal growth, rats, 334

turnover, whole-body, $\left[{ }^{15} \mathrm{~N}\right]$ glycine and $\left[1-{ }^{13} \mathrm{C}\right]$ leucine administration and, preterm infants, 381

Protein C, fetal development of, ovine fetus, 365
Pubarche children, $3 \beta$-hydroxysteroid dehydrogenase gene, molecular basis and hormonal criteria, 820

Radial artery, pressure wave, bisferiens peaks, role, marker of patent ductus arteriosus, neonates, 800

Radiant heater, water loss from skin, term and preterm infants, 233

Radioimmunoassay, IGF-I and IGF-binding protein 3, term and preterm infants, during first year, 581

RDS, see Respiratory distress syndrome

Renal disease, spontaneous nocturnal growth hormone secretion and, prepubertal children, 86

Renal failure, chronic, spontaneous nocturnal growth hormone secretion and, prepubertal children, 86

Renin-angiotensin system gene expression, cortisol effects, fetal sheep, 741

hemorrhage and, cortisol effects, fetal lambs, 316

Respiratory distress syndrome artificial ventilation effects on heart rate variability, very preterm infants, 124

surfactant apoprotein A role, plasma protein inhibitory effects on surfactant activity, 21

Respiratory failure, exogenous surfactant therapy, second dose distribution, rabbits, 476

Respiratory tract, infection, recurrent, immunoglobulin antibody responses to pneumococcal polysaccharide vaccine, 812

Retinitis pigmentosa, NARP syndrome and, clinical symptoms relation to proportion of DNA carrying point mutation, 634

Ribonucleic acid, messenger, translation regulation, gene expression control and, 681

RNA, see Ribonucleic acid

Rubella, vaccine virus, growth and passage, effects, cord blood mononuclear cells, 623

Saliva, cortisol levels through childhood and adolescence, 502

Sepsis, neonatal, interleukin-1 receptor antagonist levels in, 626

Sialyl Lewis ${ }^{\mathrm{a}}$ antigen, levels, infants and children with cystic fibrosis, 460

SIDS, see Sudden infant death syndrome

Skin

disease, leukotrienes and, biosynthesis and pathophysiologic significance, 1

fibroblasts, cultured, auto-fluorescence, mucolipidosis 4 patients, 687 surface hydrophobicity, prenatal steroids effects, preterm rats, 402

Smith-Lemli-Opitz syndrome, 3-methylglutaconic acidemia and, 671

Smooth muscle cells, migration, ductus arteriosus closure and, transforming growth factor $\beta_{1}$ inhibiting effects, fetal lamb, 561

Sodium, dietary, role, modulating atrial natriuretic peptide, neonatal rats, 310

Somatosensory, evoked potentials, brain water content and, preterm piglets after asphyxia, 661

Somatostatin, cholecystokinin and, levels, preterm infants and mother at birth, 771

Spectral analysis, heart rate variability, autonomic nervous system development and maturation and, 294

Spectroscopy

near-infrared, cytochrome $a a_{3}$ reduction analysis, role, predicting cerebral energy loss, hypoxic piglets, 253

proton magnetic resonance, brain, metabolic assessment during development, 145

proton nuclear magnetic resonance, phenylalanine levels in brain measurement, 244

Staphylococcal enterotoxin B, T cell cytokine phenotype and frequency, preterm infants, 455

Staphylococci, coagulase-negative, catheter-related infections and, 70

Staphylococcus epidermidis, virulence, catheter-related infections and, 70

Stature

idiopathic short, spontaneous nocturnal growth hormone secretion and, prepubertal children, 86

short, plasma growth hormone-binding protein and IGF-I levels, after recombinant human growth hormone therapy, Turner's syndrome patients, 106

Steroids, prenatal, skin surface hydrophobicity effects, preterm rats, 402

Stomach, development, actin and myosin levels, 202

Striatum, damage, heart injury and, umbilical cord occlusion effects, fetal sheep, 707

Sudden infant death syndrome, hypoxemia and, hyperventilatory response, maternal smoking as risk factor, 652

Surfactant

apoprotein $\mathrm{A}$, effects, plasma protein inhibitory effects, surfactant activity, 21 
SUBJECT INDEX TO VOLUME 37

exogenous, respiratory failure therapy, second dose distribution, rabbits, 476

protein B, natural surfactant and, functional effects, 271

therapy

lung vascular injury and edema effects, preterm infants, 265

pulmonary hemorrhage risk and, VLBW infant with RDS, 26

Swallowing, during apnea and periodic breathing, comparison study, preterm infants, 796

$\mathrm{T}$ cells

cytokine phenotype and frequency, preterm infants responding to staphylococcal enterotoxin B, 455

cytokine production and expression, infant versus adult, 64

Taurine transport, regulation, medium osmolality effects, 227

Thermogenesis, nonshivering, adenosine inhibiting role, fetal sheep, 303

Thrombin regulation, $\alpha_{2}$-macroglobulin and antithrombin III role, endothelial cell surfaces and, 373

Thyroid-stimulating hormone, growth-promoting actions, T-lymphoblast cell lines, 507

Thyrotropin releasing hormone, prenatal administration, lung antioxidant enzyme activity effects, fetal rats, 611

Thyroxine, maternal-fetal transfer, effects on in utero hypothyroidism, rats, 497

T-lymphoblasts, growth-promoting actions of parathyroid, adrenocorticotrophic, and thyroid-stimulating hormones, 507

TNF, see Tumor necrosis factor

Total body electrical conductivity, role, measuring body fat-free mass, infants, 94
Transplant, placental blood hematopoietic progenitor cells, hemoglobin expression after, fetus, 432

Triiodothyronine, $\beta$-adrenergic responsiveness retention, during ventricular myocyte culture, neonatal rabbits, 277

Tumor, malignant, methotrexate therapy, neurologic disorders and, biopterin and biogenic amine metabolism effects on, 151

Tumor necrosis factor

effects, neutrophil-mediated killing of nontypable Haemophilus influenzae, 155

gene, autoimmunity and, sequence analysis, 165

Turner's syndrome, plasma growth hormone-binding protein and IGF-I levels, after recombinant human growth hormone therapy, 106

Tyrosine hydroxylase, c-fos, neuropeptide mRNA and, expression in rat brain around birth, hypoxia and hypothermia effects, 15

Tyrosinemia, type I, nephropathy, after liver transplantation, 640

blood, mononuclear cells, effects, rubella vaccine virus growth and passage, 623

occlusion, striatal damage and heart injury and, fetal sheep, 707

Vaccine

pneumococcal polysaccharide, immunoglobulin antibody responses, patients with recurrent bacterial respiratory tract infections, 812
Umbilical cord rubella, virus growth and passage, umbilical cord blood mononuclear cells effects, 623

Vascular resistance, pulmonary, inhaled nitric oxide effects on, preterm lambs, 35

Vasodilation, cerebral, hypotension and, endothelial injury effects, piglets, 10

Venodilation, atrial natriuretic peptide effects, chick embryo, 465

Ventilation

artificial, effects, heart rate variability, very preterm infants, 124

surfactant therapy and, surfactant protein $B$ and natural surfactant, 271

Ventricular filling, diastolic, heart rate characteristics, chick embryo, 289

Visual development, pattern visual evoked potential evaluation, preterm infants, 140

Visual evoked potential, pattern, maturation, preterm infants, 140

Visual impairment, mucolipidosis 4 and, phenotypic marker identification, 687

Vitamin A, heart levels, alcohol ingestion during pregnancy effects, fetal alcohol syndrome and, rats, 418

Vitamin $\mathrm{D}$, deficiency, $1,25(\mathrm{OH})_{2} \mathrm{D}$ production and, rats, 726

VLBW, see Infant, very low birth weight

Water loss, skin

incubator and radiant heater, term and preterm infants, 233

prenatal steroids effects, preterm rats, 402

Wilson's disease, plasma copper and antioxidant status, 219

Zinc, absorption, metallothionein and cysteine-rich intestinal protein role, diabetic rats, 321 